\title{
NeuroAIDS in Africa
}

\begin{abstract}
Kevin Robertson ${ }^{1}$, Jeff Liner ${ }^{1}$, James Hakim ${ }^{2}$, Jean-Louis Sankalé ${ }^{3}$, Igor Grant ${ }^{4}$, Scott Letendre $^{5}$, David Clifford ${ }^{6}$, Amadou Gallo Diop ${ }^{7}$, Assan Jaye ${ }^{8}$, Georgette Kanmogne ${ }^{9}$, Alfred Njamnshi ${ }^{10}$, T. Dianne Langford ${ }^{11}$, Tufa Gemechu Weyessa ${ }^{12}$, Charles Wood ${ }^{13}$, Mwanza Banda ${ }^{14}$, Mina Hosseinipour ${ }^{15}$, Ned Sacktor ${ }^{16}$, Noeline Nakasuja ${ }^{17}$, Paul Bangirana ${ }^{17}$, Robert Paul ${ }^{18}$, John Joska ${ }^{19}$, Joseph Wong ${ }^{20}$, Michael Boivin ${ }^{21}$, Penny Holding ${ }^{22}$, Betsy Kammerer ${ }^{23}$, Annelies Van Rie ${ }^{24}$, Prudence Ive ${ }^{25}$, Avindra Nath ${ }^{26}$, Kathy Lawler $^{27}$, Clement Adebamowo ${ }^{28}$, Walter Royal III' ${ }^{29}$, and Jeymohan Joseph ${ }^{30}$ on behalf of the NeuroAIDS in Africa Conference Participants
\end{abstract}

${ }^{1}$ University of North Carolina, Chapel Hill, North Carolina, USA ${ }^{2}$ University of Zimbabwe, Harare, Zimbabwe ${ }^{3}$ Globomics, LLC, Decatur, Georgia, USA ${ }^{4}$ University of California, San Diego, La Jolla, California, USA 5 University of California, San Diego, San Diego, California, USA ${ }^{6}$ Washington University School of Medicine, St. Louis, Missouri, USA ${ }^{7}$ University of Dakar, Dakar, Senegal ${ }^{8}$ Medical Research Council Laboratories, Gambia Unit, Banjul, The Gambia ${ }^{9}$ University of Nebraska Medical Center, Omaha, Nebraska, USA ${ }^{10}$ University of Yaoundé, Yaoundé, Cameroon ${ }^{11}$ Temple University School of Medicine, Philadelphia, Pennsylvania, USA ${ }^{12}$ Addis Ababa University, Addis Ababa, Ethiopia ${ }^{13}$ Nebraska Center for Virology, University of NebraskaLincoln, Lincoln, Nebraska, USA ${ }^{14}$ University Teaching Hospital, Lusaka, Zambia ${ }^{15}$ UNC Project, Lilongwe, Lilongwe, Malawi ${ }^{16}$ Johns Hopkins University, Johns Hopkins Bayview Medical Center, Baltimore, Maryland, USA ${ }^{17}$ Makerere University, Kampala, Uganda ${ }^{18}$ University of Missouri, St. Louis, Chesterfield, Missouri, USA ${ }^{19}$ University of Cape Town, Cape Town, South Africa ${ }^{20}$ University of California, San Francisco, San Francisco, California, USA ${ }^{21}$ Michigan State University, East Lansing, Michigan, USA ${ }^{22}$ Case Western Reserve University, Cleveland, Ohio, USA ${ }^{23}$ Children's Hospital, Boston/Harvard Medical School, Newton, Massachusetts, USA ${ }^{24}$ University of North Carolina at Chapel Hill, Chapel Hill, North Carolina, USA ${ }^{25}$ Clinical HIV Research Unit, Helen Joseph Hospital, Gauteng, South Africa ${ }^{26}$ Johns Hopkins University, Baltimore, Maryland, USA ${ }^{27}$ University of Pennsylvania, Philadelphia, Pennsylvania, USA ${ }^{28}$ Office of Strategic Information and Research, Institute of Human Virology, Abuja, Nigeria ${ }^{29}$ University of Maryland School of Medicine, Baltimore, Maryland, USA ${ }^{30}$ HIV Pathogenesis, Neuropsychiatry and Treatment Branch, Center for Mental Health Research on AIDS, National Institute of Mental Health, National Institutes of Health, Bethesda, Maryland, USA

\section{Abstract}

In July 2009, the Center for Mental Health Research on AIDS at the National Institute of Mental Health organized and supported the meeting "NeuroAIDS in Africa." This meeting was held in Cape Town, South Africa, and was affiliated with the 5th IAS Conference on HIV Pathogenesis, Treatment and Prevention. Presentations began with an overview of the epidemiology of HIV in sub-Saharan Africa, the molecular epidemiology of HIV, HIV-associated neurocognitive disorders (HANDs), and HAND treatment. These introductory talks were followed by presentations on

\section{(C) 2010 Journal of NeuroVirology}

Address correspondence to Kevin Robertson, PhD, Department of Neurology, University of North Carolina, 170 Manning Drive, Chapel Hill, NC 27599-7025, USA. kevinr@neurology.unc.edu.

Declaration of interest: The authors report no conflicts of interest. The authors alone are responsible for the content and writing of the paper. 
HAND research and clinical care in Botswana, Cameroon, Ethiopia, The Gambia, Kenya, Malawi, Nigeria, Senegal, South Africa, Uganda, and Zambia. Topics discussed included best practices for assessing neurocognitive disorders, patterns of central nervous system (CNS) involvement in the region, subtype-associated risk for HAND, pediatric HIV assessments and neurodevelopment, HIV-associated CNS opportunistic infections and immune reconstitution syndrome, the evolving changes in treatment implementation, and various opportunities and strategies for NeuroAIDS research and capacity building in the region.

\section{Keywords}

HIV; neurodevelopment; neurology; neuropsychology; subtypes

\section{Introduction}

In July 2009, the Center for Mental Health Research on AIDS at the National Institute of Mental Health organized and supported the meeting "NeuroAIDS in Africa." This meeting was held in Cape Town, South Africa, and was affiliated with the 5th IAS Conference on HIV Pathogenesis, Treatment and Prevention. The goals of the meeting included:

1. Reviewing findings of neurocognitive impairment directly attributable to HIV at different African sites

2. Determining the impact of comorbid factors such as coinfections (tuberculosis [TB], malaria, syphilis, toxoplasmosis, cryptococcal meningitis, etc.), psychiatric illness, or nutritional deficiencies on neurocognitive function

3. Discussing relationships between neurocognitive impairment and HIV disease variables (current and nadir CD4, viral load, biomarkers of HIV neuropathogenesis, neuroimaging, and brain pathology)

4. Evaluating differences in HIV-1 subtype-associated neuropathology

5. Evaluating the relationships between HIV-associated neurocognitive impairment and ability to perform activities of daily living within different African populations

In addition to these goals, conference participants discussed the challenges to NeuroAIDS research in Africa as related to infrastructure, access to neurological and neuropsychological expertise, regional HIV genetic variability, host population genetics, differences in comorbidities and coinfections, availability of appropriate normative/control data for neuropsychological assessments, differences in language and culture, and differences in methods used across studies. Efforts were made at this meeting to further establish a more standardized approach to neurocognitive assessments across international studies, and expand on best practices for areas of concern such as developing appropriate normal data, identifying and categorizing levels of comorbidities, training local investigators, and assessing the relative impact of neurocognitive impairment on everyday functioning, in order to improve future NeuroAIDS research in Africa and other resource-limited settings.

\section{Overview of epidemiology and neurological disease Epidemiology of HIV/CNS disease in sub-Saharan Africa}

Dr. Hakim gave an overview of the epidemiology of HIV and central nervous system (CNS) disease in sub-Saharan Africa. According to the 2008 UNAIDS Report on the Global AIDS Epidemic, approximately 22 million (67\%) of the estimated 33 million adults and children living with HIV globally, reside in sub-Saharan Africa (WHO/UNAIDS, 2008). Of the estimated 2.7 million adults and children newly infected with HIV worldwide in 2007, 1.9 
million new infections occurred in sub-Saharan Africa as well as 1.5 million of the estimated 2 million adult and child deaths due to AIDS. Although sub-Saharan Africa is certainly still in dire straits, the 2008 UNAIDS report offered some signs of improvement. The 2008 UNAIDS Report on the Global AIDS Epidemic confirmed progress in the response to AIDS, stating that governments are acting on their promises to scale up diagnosis, prognosis, and care for HIV-infected individuals, that financing for HIV treatment in low-tomiddle-income countries has increased 6-fold and that additional countries (Lesotha, Namibia, Republic of South Africa [RSA], and Swaziland, for example) have had improvements in the epidemic (WHO/UNAIDS, 2008). CNS opportunistic infections (OIs) such as tuberculosis (meningitis, tuberculoma), cryptococcal meningitis, toxoplasmosis, neurocystercercosis, neurosyphilis, primary CNS lymphoma (Epstein-Barr virus), progressive multifocal leukoencephalopathy (JC virus), cytomegalovirus, as well as stroke remain the most commonly described HIV-associated CNS diseases. Cognitive dysfunction such as dementia and psychological perturbations unrelated to OIs above has been less commonly described. Dr. Hakim acknowledged problems with our understanding of HIVassociated CNS disease in sub-Saharan Africa, largely due to limited data on these conditions, in part due to the paucity of neurologists managing HIV-infected patients. In addition, confidently confirming diagnoses is difficult due to comorbid conditions, limited imaging facilities, and limited diagnostic capabilities (microbiological, immunological, virologic, serologic, histologic, etc.).

\section{Molecular epidemiology of HIV in Africa}

Dr. Sankalé presented a review of the molecular epidemiology of HIV. HIV exhibits tremendous genetic diversity, which has been attributed to the lack of proofreading of reverse transcriptase, rapid turnover of virions, constant recombination of viruses, RNA polymerase II errors, and selective immunologic pressures. The HIV-1 genome encodes nine open reading frames: gag, pol, env, tat, rev, nef, vpr, vif, and vpu. Major variations in these genes allow for HIV to be classified at a number of levels, beginning with distinctions between HIV-1 and HIV-2. HIV-1 consists of several major groups that include group M (Major), the source of the vast majority of HIV infections worldwide, as well as groups $\mathrm{O}$ (Outlier) and $\mathrm{N}$ (non-M, non-O). Within group $\mathrm{M}$, HIV can be further distinguished into clades or subtypes, such as clades A-D, F-H, J, K, circulating recombinant forms (CRFs) A/ $\mathrm{E}$ and $\mathrm{A} / \mathrm{G}$, many other circulating recombinant forms (which now number over 40) (LANL, 2010) as well as unique recombinant forms. The distribution of HIV-1 subtypes and recombinants varies by region of the world, by country, and even within a country such as Nigeria. Within Africa, CRF02_AG accounts for the bulk of infections in West Africa, subtypes A and D are common in East Africa, and subtype C predominates in Southern Africa as well as Northeastern areas such as Ethiopia (Hemelaar et al, 2006). Differences in disease progression and resistance profiles were also discussed. A study of registered female sex workers in Dakar, Senegal, determined that HIV-2 is less infectious, has a much lower mother-to-child transmission rate, and a much longer progression time to AIDS than HIV-1. Studies investigating differences in HIV-1 subtypes found that subtype A has a longer AIDS-free survival than non-A (Kanki et al, 1999); subtype D has a shorter time to death and AIDS than subtype A, C, or recombinant forms (Vasan et al, 2006); and in subtype C, CCR5/non-syncytia-forming viruses are almost always the dominant form even in late stages of infection (Tscherning et al, 1998). HIV-2 and HIV-1 type O possess a primary resistance to non-nucleoside reverse transcriptase inhibitors (NNRTIs) (Descamps et al, 1997; Ren et al, 2002). Naturally occurring minor mutations in the protease sequences of many non-B subtypes such as subtype F (Poveda et al, 2008) can also confer resistance. Few naturally occurring polymorphisms at resistance positions in reverse transcriptase in type $\mathrm{M}$ viruses have been discovered. 
There is a potential role for clade differences in varying neuropathogenicity. How much of a role clades play in the neuropathogenicity of HIV will be difficult to determine because treatment availability and practices vary from place to place and measures of cognitive and functional performance are inherently subject to some degree of cultural influence.

\section{HIV-associated neurocognitive disorders}

Dr. Grant gave a summary of HIV-associated neurocognitive disorders (HANDs), which remain to be common problems in HIV-infected patients despite the success of combination antiretrovirals at improving overall health and prolonging survival. The nosology of HAND, revised in 2007 (Antinori et al, 2007), now includes three classifications: asymptomatic neuropsychological impairment (abnormality in two or more cognitive abilities), mild neurocognitive disorder (cognitive impairment with mild functional impairment), and HIVassociated dementia (marked cognitive impairment with marked functional impairment). Neuropsychological (NP) deficits in measures of attention, learning, verbal, motor, memory, psychomotor, sensory, and abstraction are the most common among patients with global NP impairment. HAND prevalence rates still range from $30 \%$ to $50 \%$ and the cognitive profile, or areas of impairment, has not changed significantly. One third of patients have magnetic resonance imaging (MRI) evidence of white matter abnormality and approximately $34 \%$ (perhaps up to 60\%) have detectable HIV RNA in the cerebrospinal fluid (CSF).

The possible mechanisms for neurotoxicity in HIV-1 infection include direct damage to neurons caused by shed viral proteins, but not infection of neurons themselves, as well as indirect damage caused by neurotoxic molecules released by activated and/or infected astrocytes, macrophages, and microglia. HIV infection preferentially causes damage to the basal ganglia and white matter, but loss of dendritic complexity and the degree and synaptodendritic injury are also strongly associated with progressive neurocognitive impairment. Cofactors in HIV-associated neurocognitive complications such as drug abuse (e.g., methamphetamine), coinfections (e.g., hepatitis C), aging, immune reconstitution syndrome, neurotoxic treatments, and other background factors (e.g., head injury) tend to increase the likelihood and severity of cognitive impairment in HIV-infected individuals. On the contrary, patients with a history of good virologic control and no history of severe immunologic suppression have a reduced risk of neurocognitive impairment. In terms of everyday function, neurocognitive impairment is associated with higher rates of unemployment, difficulty with complex tasks such as driving, decreased ability to adhere to antiretroviral treatment, and increased mortality several years out from initial visit. Treatment considerations such as starting antiretrovirals (ARVs) early and including ARVs with high CSF penetration to reduce CSF viral load (VL) and prevent or attenuate HAND are promising, but still need further research.

Endeavors to establish HAND prevalence rates across countries and regions of the world have yielded varying estimates, ranging from $12 \%$ to $56 \%$. Empirical questions regarding the nature of these different rates (i.e., HIV clade differences, host responses and genetics, differences in cofactors such as TB, malaria, hepatitis, nutrition, etc.) are impeded by the lack of comparable and reliable diagnostic methods used across sites.

\section{Treatment of HAND—penetration of antiretrovirals into the CNS}

As mentioned above, HAND is still a global problem. Dr. Letendre's talk focused on the possibility that the limited distribution of drugs into the brain could be contributing to this problem. Factors limiting drug distribution into the CNS are based in the restrictive nature of the blood-brain barrier (tight junctions and astrocytic foot processes). As a result, diffusion of drugs across the blood-brain barrier is determined in part by characteristics such as protein binding, molecular weight, lipophilicity, ionization, and molecular pumps (e.g., P- 
glycoprotein and organic anion transporters). Drugs within the different classes of antiretrovirals differ in their CSF pharmacokinetics. For example, the CSF concentration of the nucleoside reverse transcriptase inhibitor (NRTI) abacavir reaches approximately $36 \%$ of plasma concentrations whereas tenofovir reaches approximately 5\%. The PIs darunavir and lopinavir differ in their CSF-to-plasma ratios (1.4\% versus $0.23 \%)$ as do NNRTIs such as nevirapine and efavirenz (29-63\% versus $0.5 \%$ ). These ratios, however, may not reflect efficacy in the nervous system as well as metrics that estimate the relationship between drug concentrations in the nervous system and the concentrations that inhibit HIV replication (e.g., the CSF-to-50\% inhibitory concentration ratio). When antiretrovirals improve or maintain cognition, they probably do so by reducing HIV replication in the CNS and improving cellular immunity. These effects are probably necessary for improving or maintaining cognitive health but may not always be sufficient because neurotoxins such as HIV proteins, proinflammatory cytokines, oxidative stress, and excitotoxins may persist even when HIV levels are reduced below the quantitation limit of commercial assays. In addition, immune recovery may not result in recovery of neurotrophic or neuroprotective molecules. The predominant cellular source of HIV found in the nervous system may determine the effectiveness of treatment on CNS complications. When CD4+ cell counts are higher and cognition is normal, HIV in the nervous system may predominantly derive from migrating cells, such as lymphocytes. In this situation, controlling HIV in migrating cells is relatively more important and penetration of antiretrovirals into the nervous system may be relatively less important. However, when CD4+ cell counts are lower or cognition is impaired, HIV in the nervous system may derive predominantly from resident cells, such as macrophages and microglia. In this situation, controlling HIV may require antiretrovirals that penetrate into the nervous system in therapeutic concentrations. Additional antiinflammatory, antioxidant, or neuroprotective therapies may also be warranted.

Dr. Letendre has devised a method of estimating the penetration and effectiveness of antiretrovirals in the nervous system. Ranks are assigned to each drug and these ranks are summed to yield a value for a given regimen, with higher scores indicating greater penetration. In most analyses to date, higher penetration ranks are associated with lower CSF viral loads. Other analyses have also identified that estimates of higher penetration are associated with better neurocognitive performance, better mood, and better survival.

\section{HAND research and clinical care in Africa}

\section{Assessment of neurological complications in HIV-2 infection in West Africa}

HIV-2 is uniquely prevalent in West Africa and although the clinical course is more indolent than HIV-1, pathological studies of brain tissue suggest similar pathology to HIV-1 by time of death. Clinical neurological manifestations of HIV-2, however, are largely unstudied. Dr. Clifford, Dr. Jaye, and Dr. Diop presented their aims for several cohorts underway to develop the infrastructure for neurological evaluation in West Africa in order to learn more about the neurological complications of HIV-2. In addition to development of infrastructure and sharing of expertise, the aims of the cohorts include (1) studying the prevalence and characteristics of neurocognitive impairment, peripheral neuropathy, and myelopathy in HIV-2-infected patients through the course of disease; (2) collecting preliminary neuroimaging and CSF samples from clinically characterized HIV-2-affected patients; (3) investigating the association of aging on HIV-2 neurological manifestations; and (4) determining the spectrum of neurologic opportunistic infections associated with HIV-2. The two cohorts discussed are based in Caio, The Gambia, and Dakar, Senegal. 


\section{NeuroAIDS in Cameroon}

Dr. Kanmogne and Dr. Njamnshi presented the preliminary results of the Cameroon neuroAIDS project, a case-control study aimed at investigating the epidemiology of HIVassociated neurocognitive disorders in Cameroon. From February 2008 to May 2009, the Cameroon team enrolled 122 participants (85 HIV-seropositive cases and 37 HIVseronegative controls). In this pilot study, both the Halstead Category Test (HCT) and Wisconsin Card Sorting Test-64 (WCST-64) showed significant impairment of the executive/frontal lobe function in HIV-infected individuals, compared to seronegative controls. Data further showed increased impairment in HIV-seropositive individuals with advanced immune deficiency.

As a baseline for a series of studies on HIV-associated dementia (HAD), Dr. Njamnshi and colleagues assessed the usefulness of the recently developed International HIV Dementia Scale (IHDS) as a screening tool for HAD or HIV-associated cognitive impairment in HIVpositive adults in Yaoundé, Cameroon. In this case-control study, HIV-positive adults followed up in an HIV outpatient clinic were matched to HIV-negative subjects for age and sex and screened using the IHDS. In particular, the IHDS mean total score, alternating hand sequence, and 4-word recall measures were useful in distinguishing between HIV patients at risk for dementia and normal healthy subjects. The IHDS fingertapping subscore was not significantly different between the two groups. Using the IHDS screening tool, investigators estimated a $21.1 \%$ prevalence rate of HAND, and suggested that future research on HAND in Cameroon should strategically start with the IHDS as a screening tool. Dr. Njamnshi and colleagues also investigated risk factors for HAND such as low hemoglobin levels, low body mass index (BMI), more constitutional symptoms before AIDS, age older than 68 at AIDS onset, intravenous (IV) drug use, low CD4 counts, high plasma viral loads, and antiretroviral (ARV) drug naivety. They found that low CD4 count $(<200 / \mu \mathrm{l})$, advanced clinical stage (Centers of Disease Control and Prevention [CDC] stage C), and low hemoglobin concentration ( $\leq 10 \mathrm{~g} / \mathrm{dl}$ ) independently predicted risk of HAND in Yaoundé, Cameroon, and suggested that these factors could be used to detect HIV-infected patients at risk of developing HAD, and included as criteria for eligibility to highly active antiretroviral therapy (HAART).

\section{Patterns of CNS involvement in the AIDS population in Ethiopia}

Utilizing postmortem forensic pathology cases in Addis Ababa, Ethiopia, Dr. Langford and Dr. Gemechu are conducting autopsies to determine the frequency of HIV infection and neuropathological patterns, as well as the frequency of CNS involvement of Mycobacterium tuberculosis in $\mathrm{HIV+}-$ - cases in this region. Investigators hypothesized that approximately $15 \%$ of cases would be HIV+, $30 \%$ to $50 \%$ of these cases would show evidence of CNS involvement, and that CNS involvement in TB+ cases would be greater in HIV+ than in HIV- cases. The design of this experiment offered opportunities to characterize the natural history of HIV in this population, observe HIV neuropathogenesis at different stages of disease, and characterize neuropathology patterns in HAART-naïve patients. Half of the cases to be considered were obtained from hospital settings and half from outside settings. Additionally, half of the cases within each group resulted from natural death, and half from injury (accidental and intentional). Gross pathological observations of 188 cases at autopsy included 112 cases (60\%) suspected of chronic illness, 37 cases (20\%) suspected of CNS infection, and 84 cases (45\%) with suspected pulmonary TB with pneumonia.

Histopathological analyses of the same 188 cases confirmed TB, encephalitis, liver disease, or other CNS-related pathologies in $42 \%$ of injury-related deaths. TB was the most common histopathological finding from both injury and natural cause groups, followed by liver disease. Tissues collected at Menelik Hospital in Addis Ababa included spleen, liver, lungs, lymph nodes, and adrenal tissue from the periphery, and frontal cortex, basal ganglia 
(lenticular nucleus), hippocampus, and spinal cord from the nervous system. Investigators noted the challenge of detecting HIV in tissue when the density of the virus may be low. As a result, tissue from the spleen and frontal cortex were sent to Temple University in Philadelphia for polymerase chain reaction (PCR) analyses. Further analyses are pending.

\section{Neuropsychological assessment in HIV/AIDS and its challenges in a low-resource country (Zambia)}

Dr. Wood and Dr. Banda spoke about their research in Zambia, one of the poorest countries in Africa. The HIV prevalence rate in Zambia is at $14 \%$ in a population estimated at 12 million. Greater than $95 \%$ of viral isolates obtained from Zambia are subtype C, and the neurological manifestations and psychological sequelae of subtype $\mathrm{C}$-infected individuals in this area are not yet known. These researchers and their collaborators are taking measures to build the necessary in-country capacity to conduct HIV-1 neuropathogenesis research to determine the impact and extent of HIV-associated neurological diseases in Zambia. As part of these aims, they conducted a pilot study among HIV- adults and children from around Lusaka to assess the utility of tools adapted from Western/developed neuropsychological assessments. Among adult controls, scores for the IHDS, Color Trails 2, Timed Gait, and Grooved Pegboard (dominant and non-dominant hand) fell below Western norms, whereas scores for the Mini-Mental State Examination and Color Trails 1 were comparable to Western norms. These results reveal that setting/culture and level/quality of education can influence neuropsychological performance, but that Western developed neuropsychological tests can be useful to assess HIV+ individuals in Zambia, when tests are adapted and performance compared to appropriate controls from the same setting.

To determine the impact and extent of HIV-associated neurological diseases in Zambia, researchers are conducting a prospective, 12-month longitudinal study, using a random sample of HIV+ and HIV- adults and children with no existing neurological or medical problems. Analysis of performance so far on the IHDS revealed that HIV+ overall performed more poorly than HIV-, but no differences between HIV+ treated and untreated individuals were indicated. Another interesting finding was that HIV+ females tended to be more sensitive to IHDS motor tasks. This ongoing study will continue to analyze other adult and child assessments up to 12 months, and this preliminary data will then be used to assess progression of neurological disease over a longer period.

Dr. Grant also spoke briefly on a pilot study in Zambia to test the feasibility of using the HIV Neurobehavioral Research Center (HNRC) International neuropsychological test battery to assess HAND in Zambia and lay the foundation for future neuroAIDS projects. Due to the current lack of Zambian test norms, US African American norms for this battery were used, with constants added to all scaled and $T$-scores in order to reduce demographic effects and allow for impairment classification via a global deficit score. Using these methods, approximately $33 \%$ of HIV+ subjects and $15 \%$ of HIV- subjects had neurocognitive impairment. Neuropsychologically impaired HIV+ subjects reported more than twice the number of cognitive complaints than those considered normal, indicating that performance relates to everyday life. The effects of HIV disease were greatest on neuropsychological domains of sensory-information processing, verbal fluency, and executive functions. The results indicate that this NP test battery shows sensitivity to HIV in Zambia where clade $\mathrm{C}$ is common in addition to its sensitivity to HIV in other international settings where clade B is common. Use of African American norms, however, only partly removed demographic effects (and only after adjustment), and Zambian norms for proper demographic correction are clearly needed in the future. 


\section{ACTG (AIDS Clinical Trials Group) 5199 International Neurological Study}

Dr. Robertson and colleagues conducted the first large-scale international neurological clinical trial in resource-limited settings for HIV. The purpose of this trial was to estimate the prevalence and incidence of AIDS dementia and CNS opportunistic infections, and study the changes in neurocognitive functioning at the initiation of antiretroviral treatment. Researchers conducted neurological and neuropsychological examinations at sites in Brazil, India, Malawi, Peru, South Africa, Thailand, and Zimbabwe to examine the effects of three treatment arms (3TC/ZDV/EFV, FTC/TDF/EFV, and FTC/ddIEC/ATV). The three sections of the neurological examination consisted of a face-to-face interview, an examination to observe the subject's physical and mental status, and a final summary section. The neuropsychological examinations included the Timed Gait Test, Finger-Tapping Test, Grooved Pegboard Test, and the Verbal Fluency Test. These neuropsychological examinations tested for fine motor function, speed of processing, gross motor function, motor control, balance, executive function, speed, and attention, The neurological and neuropsychological examinations showed a relatively high rate of peripheral neuropathy and other neurological abnormalities, but a low prevalence of minor cognitive motor disorder (MCMD) and AIDS dementia. The unexpectedly low prevalence of cognitive impairment may be a result of selection bias (higher functioning patients enrolled), which future studies should attempt to avoid. The results of the neuropsychological examinations showed the expected variations between countries, which could be due to differences in populations, cultures, HIV subtypes or resistance patterns, or variations in test administration. Overall performance on neuropsychological examinations was similar between treatment arms. Conclusions of the study indicate there were significant improvements in neuropsychological function after initiation of antiretroviral therapy in resource-limited settings, which could be contributed to the control of HIV viral load through ARV effects and/or practice effects. Dr. Robertson and colleagues are in the final stages of developing a study to collect normative comparison data at these sites to be utilized by future neuropsychological studies.

\section{Depression and HIV-associated neurocognitive deficits: Botswana pilot studies}

In the first of two pilot projects, Dr. Lawler proposed to measure the incidence of depression and dementia in Botswana, to observe the correlation between depression and cognitive test performance, to learn if performance on a screening test correlates with other cognitive measures, and to determine whether depression and/or cognitive impairment caused problems in daily activities. The tests used to measure Dr. Lawler's proposals were the International HIV Dementia Scale (IHDS), Digit Symbol-Coding Test, WHO-UCLA Verbal Learning Test, Beck Depression Inventory-Fast Screen, Prime-MD Mood Module, and Activities of Daily Living Scale (ADLs). In this study of 120 randomly selected HIV+ individuals, $24 \%$ were depressed, $38 \%$ had dementia, and approximately $10 \%$ suffered from both depression and dementia. There was a strong correlation between depression measures and incidence of depression in Botswana was consistent with other HIV studies in Africa. Furthermore, depression did not affect cognitive task performance, and it was not related to language, nationality of examiner, age, education, or CD4 count. However, depressed individuals reported more difficulty with ADLs, in particular managing medications. Performance on the screening test (IHDS) correlated with performance on other cognitive measures. This study also reported that dementia was not related to CD4 count or time since HIV diagnosis, but was related to the length of time on HAART.

The purpose of the second pilot study was to develop culturally specific assessment tools, such as the Botswana-specific Auditory Verbal Learning Test, and to compare depression and cognitive performance with well-matched groups of HIV+ and HIV- subjects. The tests used were Trails A, Digit Symbol-Coding, Botswana Auditory Verbal Learning Test, Action 
Fluency, Color Trails 2, Grooved Pegboard, Prime-MD (Depression, Anxiety, Alcohol Modules), and Activities of Daily Living Scale. This project concluded that there was no significant difference in depression between HIV+ and HIV- subjects (21\% versus $17 \%$ ), that HIV+ subjects reported more suicidal ideation $(15 \%$ versus $3 \%)$, and that there was a significant difference in anxiety between the two groups ( $42 \%$ versus $22 \%$ ). Furthermore, HIV+ subjects were significantly impaired on tests of processing speed, verbal learning and memory, fine motor speed, and executive functions. Also, subjects reported that memory was the primary reason they had difficulty adhering to medication regimens.

\section{Opportunities and challenges for NeuroAIDS research in Nigeria}

Dr. Adebamowo discussed the opportunities and challenges of NeuroAIDS research in Nigeria. Nigeria's population of 150 million and adult HIV prevalence of $2.89 \%$ afflict the nation with the second largest population of HIV+ individuals in the world. Additionally, Nigeria as a country has a low percentage of GDP (gross domestic product) spent on health care, a limited number of physicians and other care providers, and as a result only approximately $20 \%$ of adults have comprehensive correct knowledge about AIDS. In efforts to take action against the rising prevalence of HIV infection, the amount of funding from the U.S. President's Emergency Plan for AIDS Relief (PEPFAR); preventing mother to child transmission (PMTCT), increased significantly in the past 4 years, from around $\$ 10$ million to over $\$ 1.1$ billion, which led to rapid improvement of HIV counseling, screening, testing, support, and treatment. PEPFAR funding has also significantly improved the health care infrastructure throughout the country. The HIV-Nigeria ACTION Program now has 136 training centers, hub sites, ART sites, and PMTCT sites throughout the country. PEPFAR is the largest prospective cohort of HIV-infected individuals ever assembled, providing the statistical power to evaluate rare events such as neurological impairment. In addition, PEPFAR supports substantial clinical, laboratory, and informatics infrastructure, allowing record linkage and access to biological specimens. Colocation of research at PEPFAR sites provides a cost effective strategy for study of NeuroAIDS in Africa.

\section{Studies of HIV-1-related neurocognitive impairment in Nigeria}

In Nigeria, the prevalence, clinical features, and effects of treatment for HIV-related neurocognitive impairment are unknown. Dr. Royal presented a study in which treatmentnaïve subjects in Nigeria were evaluated for HIV-related neurocognitive impairment. Assessments included clinical questionnaires, general clinical laboratory testing, CD4 counts, viral load, and viral strain studies as well as previously validated cognition assessments such as the Karnofsky Performance Scale, International HIV Dementia Scale, and a detailed neurocognitive battery. Screening was critical so that the researchers could study the sole effects of HIV-induced neurological impairment rather than from other factors such as head trauma, psychiatric illness, drug use, learning disability, and active CNS infection. Conclusions drawn from this study were that these individuals had lower mean Karnofsky and IHDS scores than HIV- controls and that low IHDS scores correlated with worse clinical status. In a small subset of patients administered detailed neuropsychological testing, low effect sizes were observed on the majority of the tests. However, several tests were found to be potentially quite sensitive for detecting neurocognitive impairment in this group. Finally, analysis of the specific HIV subtypes that are found in Nigeria suggested a possible association for specific strains with the presence of cognitive impairment. Approximately 50\% of patients in Nigeria are prescribed AZT-anchored regimens, 30\% TDF-containing regimens, and approximately $20 \%$ receive regimens containing d4T. This pilot study demonstrated feasibility for performing future studies of HIV-related neurocognitive impairment in Nigeria, which includes the assessment of the effects of treatment of the disorder. 


\section{HIV clades/subtypes}

\section{Subtype-associated differences in risk for dementia in Uganda}

Investigations into subtype-associated differences in risk for HAND are currently underway throughout the world, and Dr. Sacktor and Dr. Nakasujja presented some of their research on this topic in Uganda where clades A and D are common. In a previous retrospective study of 140 HIV seroconverters in Rakai, Uganda, followed over 5 years, patients with subtype D had a faster progression to AIDS or death (Laeyendecker, 2006). Dr. Sacktor and Nakasujja followed 60 antiretroviral-naïve HIV+ individuals at risk for HIV-associated cognitive impairment in Uganda to determine if subtype D is also associated with a greater risk of dementia compared to subtype A. Individuals included in the study completed the IHDS, Grooved Pegboard, Timed Gait, WHO-UCLA Auditory Verbal Learning Test, SymbolDigit, Color Trails 1 and 2, and Digit Span Forward and Backward. Using data from the phylogenetic analysis of both $g p 41$ and gag genes, HIV+ individuals were classified as subtype $\mathrm{A}, \mathrm{C}, \mathrm{D}$, or $\mathrm{A} / \mathrm{D}$ recombinants.

Results indicate that HIV subtype D is associated with an increased risk of dementia compared to subtype A. Of the HIV+ individuals with subtype D, $89 \%$ were categorized with dementia (MSK 1), whereas only $24 \%$ of individuals with subtype A received this rating. These results provide the first evidence in well-characterized HIV+ patients at a similar stage of HIV disease, suggesting that HIV subtypes may have different biological properties with respect to their capacity to cause HIV-associated cognitive impairment.

\section{Neuropsychological profile of patients commencing HAART in Cape Town, South Africa- preliminary findings}

Subtype C is responsible for more than $90 \%$ of HIV-1 infections in South Africa and may differ from subtype B in terms of protein binding sites, binding characteristics, replicative capacity, and possibly in neurotoxicity and risk for cognitive impairment. Dr. Joska, Dr. Paul, and colleagues discussed a natural mutation in the TAT protein in subtype $\mathrm{C}$ associated with reduced monocyte chemokine regulation and its relevance to the CNS. TAT is involved in the migration of monocytes into the brain via up-regulation of inflammatory cytokines and adhesion molecules. TAT also disrupts the tight junctions in the blood-brain barrier (BBB), therefore variations in the TAT protein could lead to variations in CNS pathology.

An early report from Ethiopia suggested that HAD is infrequent in clade C (Clifford et al, 2007), but studies from India suggest that it is comparable to clade B (Gupta et al, 2007). Dr. Joska, in collaboration with the University of Missouri, conducted a pilot study to determine if cognitive impairment exists in clade C HIV in South Africa and to begin to define neuroimaging biomarkers of CNS involvement. Cognitive measures used included Hopkins Verbal Learning Test-R, Brief Visual Memory Test-R, Stroop Test, Color Trails, Digit Symbol, Grooved Pegboard, Tapping, and Animal Fluency. Participants included 52 ARTnaïve HIV patients (29.6 years of age; 9.7 years of education) and 25 controls (27.0 years of age; 11.3 years of education). HIV patients performed significantly worse on the measures of Digit Symbol, Color Trails 2, Animal Fluency, and Stroop Color-Word interference. Analysis of diffusion tensor imaging (DTI) and fractional anisotropy also revealed significant differences between these groups. These preliminary results provide evidence for cognitive impairment in HIV clade C infection in South Africa, and these investigators plan to determine if cognitive status is associated with a TAT defect and whether or not relationships exist between cognitive status and proviral DNA, inflammatory cytokines, and neuroimaging. 


\section{Variation in neurocognitive impairment based on HIV-1 subtype}

A discussion of the effect of HIV-1 subtype on neurocognitive impairment in pediatric infection was presented by Dr. Boivin, Dr. Wong, and Mr. Bangirana. These researchers sought to determine whether HIV+ school-aged children in Uganda currently ineligible for ART demonstrate deficits relative to HIV- children and whether the risk for progressive encephalopathy (PE) in children differs by subtype.

Subtype, along with other clinical indicators, may be an important consideration for when to initiate ART treatment and for antiretroviral selection (e.g., CNS penetration characteristics). One hundred and two HIV-infected antiretroviral therapy (ART)-naïve children (mean age $=8.8$ years) and 104 noninfected controls (mean $=8.9$ years) completed the Kaufman Assessment Battery for Children, 2nd edition (KABC-2), Tests of Variables of Attention (TOVA) auditory and visual tests, and the Bruininks/Oseretsky Test for Motor Proficiency, 2nd edition (BOT-2). HIV-1 subtype was defined by the composition of the env gene determined using a sequence-specific real time PCR assay (Hoelscher et al, 2002). Evaluation of performance demonstrated significant differences between the HIV+ and HIV - groups on the $\mathrm{KABC}$ measures of memory, learning, and visual-spatial tasks, visual TOVA response time, and attention-deficit hyperactivity disorder (ADHD) score and on the BOT's manual dexterity, upper-limb coordination, and speed and agility tests. There were no differences between HIV+ and HIV- children on auditory TOVA. A comparison of HIV + children with subtypes A and D indicated subtype A children did more poorly than subtype D on visual-spatial tasks and had more errors on visual TOVA. No differences were reported between HIV+ children with subtype A versus subtype D on the BOT and auditory TOVA.

In summary, ART-naïve HIV+ Ugandan school age children have poorer neuropsychological performance on working memory, visual-spatial processing, ADHD index, and aspects of motor development. HIV subtype A is associated with poorer neuropsychological performance (visual spatial processing and TOVA impulsivity), compared to subtype D in treatment-naïve Ugandan children. HIV subtypes A and D may be associated with different risks for progressive encephalopathy (PE) in children. HIV subtype influences on encephalopathy may fundamentally differ between children and adults as subtype D was associated with a greater risk of dementia or HAND compared to subtype A in Ugandan adults. The PE described here in children may be less dependent on immune suppression but instead may be influenced by HIV coreceptor use. According to their pathogenic model of subtype differences, subtype A, which is more CCR5-tropic, may be more efficient than subtype D, which is CXCR4-tropic at infecting CNS macrophage and microglia cells. X4 virus may be more immunologically pathogenic because it infects a wider range of cells in lymphoid tissue, but may replicate less efficiently in the CNS.

\section{Pediatric Neuroassessments in Africa}

In 2007, an estimated 370,000 children became newly infected with HIV. Over $90 \%$ of these infections occurred in sub-Saharan Africa (WHO/UNAIDS, 2008). More than $90 \%$ of new infections in children occur vertically, during pregnancy, at time of birth, or through breastfeeding (WHO/UNAIDS, 2008). HIV infection in vertically infected children impairs the development and growth of an immature CNS, resulting in delayed acquisition of neurodevelopmental milestones in the majority of children in the pre-ART era (Diamond et al, 1987; Epstein et al, 1986). Without treatment, approximately one half of children will die by 2 years of age (Newell et al, 2004).

Neurocognitive assessments in HIV-infected children, especially in Africa, are plagued by a long list of confounding factors, which include the socioeconomic status or quality of the 
home environment, the emotional well-being of caregivers (i.e., depression), and secondary effects of HIV such as malnutrition, quality of education, quality of prenatal care, and treatment with antiretroviral drugs.

\section{Neurobehavioral development in a pediatric population exposed to HIV}

HIV affects all domains of child functioning at least to some extent. Motor development is the most affected in term of severity, early onset, and persistence across the age groups. Dr. Holding and colleagues investigated the role of weight-for-age and disease stage in poor psychomotor outcome of HIV-infected children in Kilifi, Kenya. They used a locally developed and validated measure, the Kilifi Developmental Inventory, to assess psychomotor development in 48 children aged 6 to 35 months prenatally exposed to HIV (exposed and infected odds ratios [OR] versus exposed and not infected). Investigators reported a significant main effect of HIV status $(F(2,38.01)=7.89, P<001)$. Children in the HIV-infected group had lower mean psychomotor scores than both exposed but uninfected children and controls. No significant differences were observed between the HIV-exposed group and the reference population. Disease stage had an inverse relationship with psychomotor outcome. Mean psychomotor scores by disease stage were stage $1,0.40$ (1.68); stage 2, -1.01 (1.93); stage 3, -1.46 (1.57). Children in disease stages 2 and 3 differed significantly from those of the reference population. Weight-for-age had a positive relationship with psychomotor outcome. These findings indicate that weight-for-age and disease stage are viable, easily measurable benchmarks to identify those children at developmental risk. These findings also imply that nutritional intervention and other measures aimed at slowing down disease progression may delay the onset and severity of psychomotor impairment in the paediatric HIV population in Africa.

\section{Effects of HIV and ARV exposure on child health and neurodevelopment, Botswana}

Perinatal ARV medications have led to dramatic reductions in mother-to-child transmission (MTCT) of HIV-1. One third of HIV+ pregnant women now receive ARVs during pregnancy or labor, and HAART use during pregnancy is increasing. Concerns related to possible negative effects of prenatal ARV exposure on neurodevelopment have been raised, given the unknown impact of ARVs on the developing brain and issues of potential mitochondrial toxicity related to NRTI exposure. A recent large well-controlled study showed no cognitive or motor differences between ARV-exposed and -unexposed HIVchildren (Williams et al, 2010). HIV-exposed but uninfected children have also shown poorer outcomes than HIV unexposed children (Van Rie et al, 2008). Studies of neurological and neurodevelopmental outcomes in HIV/ARV-exposed but uninfected children have yielded conflicting results and controlling for confounding factors (access to care, substance abuse, socioeconomic status [SES]) is problematic.

Dr. Kammerer, Dr. Lockman, and colleagues are currently working on a project in Botswana to further investigate the effects of ARV and HIV exposure in children. Botswana has the second highest rate of HIV in the world and infection affects all socioeconomic levels. In addition, $33.7 \%$ of pregnant women are HIV+, but significant government support has led to $>90 \%$ of pregnant women being tested for HIV and $>85 \%$ of HIV+ women taking ARVs. The primary objectives of the current study are (1) to compare neurodevelopmental outcomes following in utero exposure to three-drug HAART (two or more NRTIs and single NRTI [zidovudine]) among HIV-uninfected children born to HIV+ mothers (with similar CD4 cell counts); (2) to prospectively compare neurodevelopmental outcomes and rates of mortality; and (3) to determine the biologic and social factors most strongly associated with excess mortality and neurodevelopmental delay between children who are HIV exposed but uninfected and children born to HIV-uninfected mothers in a setting with HAART availability. Neurodevelopmental outcomes will be measured by a combination of an 
adapted US-normed test (Bayley Scales) that is used in comparable studies and tests developed and normed in Africa (Abubakar et al, 2008). Maternal infection status, mental health, physical health, social support, and nutrition status will be assessed as well as form of feeding (breastfeeding versus formula), health and nutrition, vision, hearing, and neurological status.

\section{Effects of HIV on the neurodevelopment of young infants in Africa: experience from Malawi}

Dr. Van Rie discussed her work on the effects of HIV and ART on neurodevelopment of infants and children in Africa. In Kinshasa, Democratic Republic of Congo, Van Rie and colleagues concluded that the vast majority of HIV-infected children, even young children, present with severe motor and cognitive developmental delay at time of diagnosis (Van Rie et al, 2008). Access to HIV care accelerated motor development, but did not result in a catch up of cognitive development. Timing of care had no effect on motor development but the cognitive development of children who accessed care early (prior to development of severe immunodeficiency) tended to normalize within 1 year of care, whereas the cognitive development remained delayed for those presenting late (Van Rie et al, 2009). These findings underscore the importance of early treatment of HIV infection in children. Dr. Van Rie is currently studying the effects of early ART (within the first 3 to 4 months of life) on neurodevelopment in Blantyre, Malawi. Preliminary data indicate that children who start treatment early may demonstrate neurodevelopmental delay (both motor and cognitive) at a very early stage in life (age 3 months) but can achieve a motor and cognitive development similar to that of HIV unexposed children by 6 months of age.

\section{Overview of treatment issues}

Implementing HIV treatment in Africa faces many challenges such as a high disease burden, many countries with poor health infrastructure, ensuring quality of care with trained staff and appropriate monitoring tests, getting people into care before disease progression, dual epidemics of TB and HIV, providing affordable and convenient regimens with favorable toxicity profiles, and sustainability of funding for ART programs. In spite of these challenges many countries are making progress in getting ART to those in need. From the years 2004 to 2007, South Africa, Uganda, Malawi, and Ghana, for example, drastically increased the estimated number of people receiving ART; however, only $27 \%, 33 \%, 35 \%$, and $15 \%$ of the estimated number of people needing ART were receiving medication based on UNAIDS/WHO criteria.

\section{Evolving changes of treatment implementation in Africa}

Dr. Ive reviewed the challenges and changes in HIV treatment in Africa and discussed a 4year program called the NSP implemented in South Africa in 2007. The NSP aims to (1) reduce the number of new HIV infections by $50 \%$ and (2) reduce the impact of HIV and AIDS on individuals, families, communities, and society by expanding access to appropriate treatment, care, and support to $80 \%$ of all people diagnosed with HIV. In particular, young people in the age group 15-24 are a focus of all the interventions, especially for behavior change-based prevention. The interventions needed to reach the goals of the NSP include (1) prevention; (2) treatment, care, and support; (3) human and legal rights; and (4) monitoring, research, and surveillance. Training and sustaining valuable human resources (i.e., doctors and nurses) is one of the biggest obstacles to providing quality care. In areas where doctors are scarce, governments and care providers may need to consider "task shifting," and delegate some activities to less qualified personnel. Training of primary health care nurses (rather than doctors) to initiate antiretroviral treatment, lay counsellors (rather than nurses) "pricking" patients for rapid HIV tests, and lay counsellors (instead of social 
workers) for orphan support activities, for example, may save time and money. Another pitfall of treatment programs in Africa is "stock outs" of antiretroviral drugs in general or specific components of a drug regimen. Stock outs are problematic because they undermine the message of adherence, increase the chance of resistance to first line regimens, and delay putting patients onto therapy.

Two important changes in HIV clinics in Africa involve the use of $\mathrm{d} 4 \mathrm{t}$ and viral load monitoring. From 2000 to 2004, d4t was a staple of ART in Africa because it was effective and inexpensive. Long-term use of the drug, however, was associated with a significant number of adverse events (e.g., peripheral neuropathy and lactic acidosis), which led to nonadherence or necessitated a change in regimen. As a result, $\mathrm{d} 4 \mathrm{t}$ is now phasing out of treatment. Monitoring viral load on the other hand is becoming more common. Although it was once believed to be too expensive, the costs of late detection of treatment failure and resulting high levels of drug resistance now overshadow the costs of monitoring viral load.

\section{HIV-associated CNS opportunistic infections and immune reconstitution syndrome}

Immune reconstitution inflammatory syndrome (IRIS) is a paradoxical worsening of a patient's clinical condition that is attributable to the recovery of the immune system after initiation of HAART. IRIS occurs in $15 \%$ to $25 \%$ of patients on HAART and in $20 \%$ to $45 \%$ of patients with OIs on HAART (Shelburne et al, 2006). Approximately 50\% of patients that develop IRIS have been on HAART for around 50 days but the syndrome can appear earlier in treatment. Patients who are antiretroviral naïve, have active or subclinical OIs at HAART initiation, have CD4 count $<50$ cells $/ \mathrm{mm}^{3}$, or have a prompt decrease in viral load with HAART are at greater risk for IRIS. Certain host genes can also make a patient more susceptible. CNS infections associated with IRIS include viral infections (JC virus: primary multifocal leukoencephalopathy [PML]; varicellazoster virus [VZV]: vasculitis; cytomegalovirus [CMV]: retinitis; Epstein-Barr virus [EBV], optic neuropathy and primary CNS lymphoma), bacterial infections (mycobacterial disease: TB, leprosy), fungal infections (cryptococcal meningitis, candida meningitis), and parasitic infections (toxoplasmosis). Encephalitis directly attributable to HIV also occurs.

Dr. Nath presented three different cases showing the spectrum of IRIS that clinicians encounter, as well as a review of the histopathological correlates, potential biomarkers, and treatment options for IRIS. In the first case, a patient with cryptococcal meninigitis on HAART and treatment for cryptococcal infection for 8 months developed headache, photophobia, and nausea while their CD4 count increased from 24 to 124 cells $/ \mathrm{mm}^{3}$ and their viral load dropped to undetectable levels. After treatment with dexamethasone $24 \mathrm{mg} /$ day, the patient dramatically improved and was diagnosed with IRIS with cryptococcal meningitis (Venkataramana et al, 2006). In the second case, a patient with a CD4 count of 474 and HIV viral load <200 developed an episode of aphasia and seizures after 10 years of HAART. In this case the dementia was mediated by macrophages in the CNS. The patient temporarily improved with steroids but then encountered more cognitive and motor issues (Venkataramana et al, 2006). The last was a case of acute encephalopathy with IRIS. A patient developed right side weakness and cognitive decline over a period of 8 weeks and then started HAART. Ten weeks later, the CD4 count improved from 50 to 320 and the viral load dropped from 838,100 to 4000 , but then over the course of another week the patient progressively declined with focal and generalized seizures resulting in death with complications this time attributed to CD8+ T cells (Miller et al, 2004).

IRIS associated with progressive multifocal leukoencephalopathy (PML) is characterized by CD3+ T cell and macrophage (CD68) infiltration, infection of oligodendrocytes by JC virus, and inflammation attributed mostly to perivascular CD8+ T lymphocytes, whereas CD4+ lymphocytes are absent. Neuropathological findings include multiple demyelinating foci in 
frontal, parietal, and temporal white matter (mostly subcortical areas). Potential biomarkers for IRIS include cytokines (e.g., interleukin-6 [IL-6]) and markers of neuronal injury (axonal and synaptic proteins). IRIS usually onsets after several weeks of HAART when the viral load drops and CD8+ T lymphocytes spike, then progresses as CD4+ T lymphocytes increase and CD8 cells decline. When managing potential CNS-associated IRIS, clinicians must first exclude effects of drugs, nonadherence to drugs, and progression of underlying disease. Then they must decide whether to discontinue HAART and whether to give systemic corticosteroids (Riedel et al, 2006). The treatment options for IRIS are not ideal because steroids increase risks from immune suppression and interruption of HAART increases risk for resistance to therapy and reemergence of IRIS upon resumption of HAART. The current recommendations for use of steroids in IRIS are high-dose steroids tapered with oral steroids for a month (with OI prophylaxis) for catastrophic IRIS, but the same treatment for symptomatic and asymptomatic IRIS is debatable.

\section{Discussion}

Although research and treatment of HIV-associated neurocognitive disorders has been going on for over 20 years in the developed world, much less has been carried out in resourcelimited setting (RLS) such as Africa. Participants at the conference discussed some of the gaps in the knowledge and care of HAND in Africa and offered several ideas to work towards bridging the gaps. One concern was that HIV care providers in Africa may not have the same general level of awareness of HAND or neuroAIDS and a network to spread general information on the disease may help. In addition to this need to spread general information on HAND, forming working consortiums and cooperative arrangements between research groups were also of great interest. Participants discussed establishing these arrangements in order to share resources on tissue samples, neuropsychological data and tools, and neuroimaging amongst other needs and considered modeling the consortium on the Institutional Development Award (IDeA) program, which extends NIH resources to foster health-related research in rural and medically undeserved areas of the US. Possible arrangements could include collaboration building between medical schools and institutions in the United States/Europe and RLS as well as site-to-site (or south-to-south) sharing of expertise. If research sites with adequate expertise can be identified, the field of HAND research in Africa could benefit from networks developed on the continent, which would have less overwhelming differences between sites. Before these efforts can move forward, however, a concrete set of training needs and resources needs to be established.

Establishing a set of neurological screens, neuropsychological examinations, and functional assessments that can be used across sites, however, is a problem on its own. Even within Africa, differences in culture, language, education, and access to resources exist, which could affect performance, interpretation, and validity of many instruments.

The issue of adapting, validating, and gathering normative data for neuropsychological assessments occupied much of the discussion section. Is it possible to develop and validate a set of tests that can be used across cultures? Most participants agreed that many instruments developed in Western setting can be used in RLS, if properly adapted for education level and culture of the setting; however, they also established that developing one set of instruments to be used across sites is not the best way to screen for neurocognitive impairment. Instead, developing a set of domains to be tested, in place of a strict set of tests, may be useful. It may not be possible to test each domain in every setting due to restraints on time, staff, and multiple issues with language proficiency and culture. Starting with a breadth of assessments and working to develop a set of domains for each setting might be better than creating many tests from scratch or using the same tests across settings. To lay the foundation for HIV neurological studies in RLS, the ACTG 5199 International 
Neurological Study selected a brief battery of neuropsychological tests sensitive to HIVassociated changes and also geared towards limiting cultural influences. In addition, researchers at these sites will soon begin a normative control study with a slightly expanded battery, aiming to enroll approximately 2400 participants, in order to address the lack of normative data in RLS.

The appropriateness of activities of daily living scales and other functional assessment also came into question. In RLS, where most people are unemployed and don't drive cars, and where work and daily life are less complex, thus many existing instruments may not be useful to determine functional impairment. The Bolton questionnaire developed in Uganda represents an improvement in this area, but developing other culturally appropriate functional assessments, possibly with help from community members, presents an ideal research project.

Other problems that occur with neuropsychological instruments and neurological examinations involve standardized administration, scoring, and definitions of clinical outcomes. Cultural differences in administration and interpretation of instructions exist, so quality control to measure performance of those giving assessments should be in place, and methods for monitoring quality should be published with research findings. Experimenter drift (away from standardized assessment) is common in both developed and developing settings, and must be managed. Primary investigators should consider observing multiple testing sessions every few months, even if they don't know the language. Reviewing CRFs and rescoring neuropsychological tests randomly can also help catch mistakes and reveal systematic errors. Periodically reviewing training DVDs that model appropriate testing techniques and blinding people administering assessments as much as possible to the disease status of the individual may also benefit standard administration. Regarding clinical outcomes, depression, functional impairment, and cognitive impairment are defined in different ways by different studies, which create issues with comparing performance and health across populations. The "Frascati paper" in 2007 provided an update of the research nosology for HAND and laid out a set of suggested cognitive domains and definitions for cognitive and functional impairment (Antinori et al, 2007). The key, however, may not be a set of definitions, but rather for researchers to be very specific when presenting findings as to what definitions were used to define cognitive/functional/behavioral impairment, and to point out differences in those definitions from other studies.

There are many challenges to conducting neuropsychological assessments in pediatric populations in Africa. Quality of home environment, physical and mental well-being of caregivers, gestational effects (MTCT and prenatal treatment), and malnutrition may all impact performance. Participants discussed different strategies for evaluating neurocognition in children, which included screening versus in-depth assessments, home- or village-based evaluations when clinic access is difficult, use of computers and cutting-edge developments, as well as strategies for standardizing scores. One proposal was to use reference groups of children based on age, gender, and environment instead of large normative data sets. Developing assessments appropriate to cultural contexts that are sustainable (do not involve published/copyrighted materials) and then determining appropriate developmental milestones for assessments in resource-limited settings were also discussed.

Neuropsychological "interventions" to boost positive neuroplasticity, such as games for learning and development in school-age HIV+ children, may compliment ART and help ameliorate some of the neurocognitive effects of HIV.

In terms of neuropathogenesis, several areas were considered. As older, better penetrating ARVs are gradually replaced by newer drugs, some unintended loss of neurocognitive benefits may occur. The effects of HIV-2 dual infection on neurocognition, as well as the 
other opportunistic infections and cofactors such as crystal methamphetamine abuse in South Africa, were also of concern. Linking systemic immunological activation and symptoms to CNS immune activation and clarifying the clade- or subtype-related mechanisms for disease need further attention. For example, in adults, clade D demonstrates increased neuropathogenesis over clade A, whereas the opposite effect was observed in children. Studies investigating clade $\mathrm{C}$ have reported less motor effects than other clades. These and other studies need to be replicated to determine if clade-specific neurocognitive profiles exist. Studies such as these are difficult to interpret, however, because even within clades, different motifs and protein products exist. The differences in pathogenesis may not be due to clades per se, but specific protein motifs within a genome that cause toxicity. Dr. Nath demonstrated that even a single amino acid shift can make a difference. If serine is substituted for cysteine, the TAT protein is less toxic because the cysteine on TAT hooks in with cysteine on a receptor to open a channel. Post-translational modifications of proteins such as TAT within mammalian cells make them 1000 times more toxic than TAT produced from Esherichia coli. Ultimately determining the mechanisms behind clade-related differences in neurocognitive impairment will be very complicated and require a lot of sequencing data in addition to neurocognitive assessments and analyses of other variables.

\section{Conclusions}

NeuroAIDS research in Africa faces many challenges, but also offers many opportunities. Because sub-Saharan Africa was the "ground zero" for the HIV pandemic, the majority of HIV-infected individuals reside there. If the effects of culture, language, and education can be teased out, this large pool of prospective patient populations presents opportunities to investigate how differences in viral and host genetics influence clinical presentation of neurocognitive disease. In addition, collections of postmortem tissue samples acquired in the region could be valuable resources for further characterizing the neuropathogenesis of HIV and comorbid conditions. The population of the region is generally resource poor and at high risk of disease, so access to treatment and counseling provided by research is very meaningful. Knowledge gained from HAND research in the region could significantly improve HAND treatment throughout the world.

\section{Complete list of conference participants}

Speakers and chairpersons

Clement Adebamowo (Abuja, Nigeria)

Mwanza Banda (Lusaka, Zambia)

Paul Bangirana (Kampala, Uganda)

Michael Boivin (East Lansing, USA)

David Clifford (St. Louis, USA)

Amadou Gallo Diop (Dakar, Senegal)

Graham Fieggen (Cape Town, South Africa)

Igor Grant (San Diego, USA)

James Hakim (Harare, Zimbabwe)

Jeannine Heckmann (Cape Town, South Africa) 
Penny Holding (Mombasa, Kenya)

Mina Hosseinipour (Lilongwe, Malawi)

Prudence Ive (Gauteng, South Africa)

Assan Jaye (Banjul, The Gambia)

Jeymohan Joseph (Bethesda, USA)

John Joska (Cape Town, South Africa)

Betsy Kammerer (Newton, USA)

Georgette Kanmogne (Omaha, USA)

T. Dianne Langford (Philadelphia, USA)

Kathy Lawler (Philadelphia, USA)

Scott Letendre (San Diego, USA)

Jeanne McDermott (Bethesda, USA)

Marc Mendelson (Cape Town, South Africa)

Noeline Nakasujja (Kampala, Uganda)

Avindra Nath (Baltimore, USA)

Alfred Njamnshi (Yaoundé, Cameroon)

Robert Paul (Chesterfield, USA)

Kevin Robertson (Chapel Hill, USA)

Walter Royal III (Baltimore, USA)

Ned Sacktor (Baltimore, USA)

Jean-Louis Sankalé (Decatur, USA)

Soraya Seedat (Cape Town, South Africa)

Annelies Van Rie (Chapel Hill, USA)

Tufa Gemechu Weyessa (Addis Ababa, Ethiopia)

Joseph Wong (San Francisco, USA)

Charles Wood (Lincoln, USA)

Participants

Jing Bao (Bethesda, USA)

Kathleen Bateman (Cape Town, South Africa) 
Mariana Cherner (San Diego, USA)

Ronald Collman (Philadelphia, USA)

Marc Combrinck (Cape Town, South Africa)

Luminita Ene (Bucharest, Romania)

Edwin Escobar G. (Miranda, Venezuela)

Nathan Geffen (Cape Town, South Africa)

Karl Goodkin (Los Angeles, USA)

Hetta Gouse (Cape Town, South Africa)

W. David Hardy (Los Angeles, USA)

Jacqueline Hoare (Cape Town, South Africa)

Jennifer Jardine (Cape Town, South Africa)

Cecilia Kanyama (Cape Town, South Africa)

Johnstone Kumwenda (Blantyre, Malawi)

Barbara Laughton (Tygerberg, South Africa)

Jeff Liner (Chapel Hill, USA)

Suzaan Marais (Cape Town, South Africa)

Ernesta M. Meintjes (Cape Town, South Africa)

Rosie Mngqibisa (Durban, South Africa)

Agnes Moses (Lilongwe, Malawi)

Daphne Radebe (Gauteng, South Africa)

Ian Sanne (Johannesburg, South Africa)

Fabienne Shumbusho (Kigali, Rwanda)

Lawrence Lee Soon-U (Singapore)

Stephen Ssebulime (Kampala, Uganda)

Kevin Stoloff (Cape Town, South Africa)

Helen Struthers (Diepkloof, South Africa)

Innocent Turate (Kigali, Rwanda)

Victor G. Valcour (San Francisco, USA)

Moleen Waison (Harare, Zimbabwe) 


\section{References}

Abubakar A, Holding P, van Baar A, Newton CR, van de Vijver FJ. Monitoring psychomotor development in a resource-limited setting: an evaluation of the Kilifi Developmental Inventory. Ann Trop Paediatr. 2008; 28:217-226. [PubMed: 18727851]

Antinori A, Arendt G, Becker JT, Brew BJ, Byrd DA, Cherner M, Clifford DB, Cinque P, Epstein LG, Goodkin K, Gisslen M, Grant I, Heaton RK, Joseph J, Marder K, Marra CM, McArthur JC, Nunn M, Price RW, Pulliam L, Robertson KR, Sacktor N, Valcour V, Wojna VE. Updated research nosology for HIV-associated neurocognitive disorders. Neurology. 2007; 69:1789-1799. [PubMed: 17914061]

Clifford DB, Mitike MT, Mekonnen Y, Zhang J, Zenebe G, Melaku Z, Zewde A, Gessesse N, Wolday D, Messele T, Teshome M, Evans S. Neurological evaluation of untreated human immunodeficiency virus infected adults in Ethiopia. J NeuroVirol. 2007; 13:67-72. [PubMed: 17454450]

Descamps D, Collin G, Letourneur F, Apetrei C, Damond F, Loussert-Ajaka I, Simon F, Saragosti S, Brun-Vezinet F. Susceptibility of human immunodeficiency virus type 1 group $\mathrm{O}$ isolates to antiretroviral agents: in vitro phenotypic and genotypic analyses. J Virol. 1997; 71:8893-8898. [PubMed: 9343254]

Diamond GW, Kaufman J, Belman AL, Cohen L, Cohen HJ, Rubinstein A. Characterization of cognitive functioning in a subgroup of children with congenital HIV infection. Arch Clin Neuropsychol. 1987; 2:245-256. [PubMed: 14589616]

Epstein LG, Sharer LR, Oleske JM, Connor EM, Goudsmit J, Bagdon L, Robert-Guroff M, Koenigsberger MR. Neurologic manifestations of human immunodeficiency virus infection in children. Pediatrics. 1986; 78:678-687. [PubMed: 2429248]

Gupta JD, Satishchandra P, Gopukumar K, Wilkie F, Waldrop-Valverde D, Ellis R, Ownby R, Subbakrishna DK, Desai A, Kamat A, Ravi V, Rao BS, Satish KS, Kumar M. Neuropsychological deficits in human immunodeficiency virus type 1 clade C-seropositive adults from South India. J NeuroVirol. 2007; 13:195-202. [PubMed: 17613709]

Hemelaar J, Gouws E, Ghys PD, Osmanov S. Global and regional distribution of HIV-1 genetic subtypes and recombinants in 2004. AIDS. 2006; 20:W13-W23. [PubMed: 17053344]

Hoelscher M, Dowling WE, Sanders-Buell E, Carr JK, Harris ME, Thomschke A, Robb ML, Birx DL, McCutchan FE. Detection of HIV-1 subtypes, recombinants, and dual infections in east Africa by a multi-region hybridization assay. AIDS. 2002; 16:2055-2064. [PubMed: 12370505]

Kanki PJ, Hamel DJ, Sankale JL, Hsieh C, Thior I, Barin F, Woodcock SA, Gueye-Ndiaye A, Zhang E, Montano M, Siby T, Marlink R, I ND, Essex ME, S MB. Human immunodeficiency virus type 1 subtypes differ in disease progression. J Infect Dis. 1999; 179:68-73. [PubMed: 9841824]

Laeyendecker, O.; Li, X.; Arroyo, M.; McCutchan, F.; Gray, R.; Wawer, M.; Serwadda, D.; Nalugoda, F.; Kigozi, G.; Quinn, T. Rakai Health Science Program. 13th Conference on Retroviruses and Opportunistic Infections; February 5-February 8, 2006; Denver, CO.

LANL. HIV Sequence Database. Los Alamos, NM: Los Alamos National Laboratory; 2010. The circulating recombinant forms (CRFs).

Miller RF, Isaacson PG, Hall-Craggs M, Lucas S, Gray F, Scaravilli F, An SF. Cerebral CD8+ lymphocytosis in HIV-1 infected patients with immune restoration induced by HAART. Acta Neuropathol. 2004; 108:17-23. [PubMed: 15085359]

Newell ML, Coovadia H, Cortina-Borja M, Rollins N, Gaillard P, Dabis F. Mortality of infected and uninfected infants born to HIV-infected mothers in Africa: a pooled analysis. Lancet. 2004; 364:1236-1243. [PubMed: 15464184]

Poveda E, de Mendoza C, Parkin N, Choe S, Garcia-Gasco P, Corral A, Soriano V. Evidence for different susceptibility to tipranavir and darunavir in patients infected with distinct HIV-1 subtypes. AIDS. 2008; 22:611-616. [PubMed: 18317002]

Ren J, Bird LE, Chamberlain PP, Stewart-Jones GB, Stuart DI, Stammers DK. Structure of HIV-2 reverse transcriptase at 2.35-A resolution and the mechanism of resistance to non-nucleoside inhibitors. Proc Natl Acad Sci USA. 2002; 99:14410-14415. [PubMed: 12386343] 
Riedel DJ, Pardo CA, McArthur J, Nath A. Therapy insight: CNS manifestations of HIV-associated immune reconstitution inflammatory syndrome. Nat Clin Pract Neurol. 2006; 2:557-565. [PubMed: 16990829]

Shelburne SA, Montes M, Hamill RJ. Immune reconstitution inflammatory syndrome: more answers, more questions. J Antimicrob Chemother. 2006; 57:167-170. [PubMed: 16354748]

Tscherning C, Alaeus A, Fredriksson R, Bjorndal A, Deng H, Littman DR, Fenyo EM, Albert J. Differences in chemokine coreceptor usage between genetic subtypes of HIV-1. Virology. 1998; 241:181-188. [PubMed: 9499793]

Van Rie A, Dow A, Mupuala A, Stewart P. Neurodevelopmental trajectory of HIV-infected children accessing care in Kinshasa, Democratic Republic of Congo. J Acquir Immune Defic Syndr. 2009; 52:636-642. [PubMed: 19730268]

Van Rie A, Mupuala A, Dow A. Impact of the HIV/AIDS epidemic on the neurodevelopment of preschool-aged children in Kinshasa, Democratic Republic of the Congo. Pediatrics. 2008; 122:e123-e128. [PubMed: 18595957]

Vasan A, Renjifo B, Hertzmark E, Chaplin B, Msamanga G, Essex M, Fawzi W, Hunter D. Different rates of disease progression of HIV type 1 infection in Tanzania based on infecting subtype. Clin Infect Dis. 2006; 42:843-852. [PubMed: 16477563]

Venkataramana A, Pardo CA, McArthur JC, Kerr DA, Irani DN, Griffin JW, Burger P, Reich DS, Calabresi PA, Nath A. Immune reconstitution inflammatory syndrome in the CNS of HIV-infected patients. Neurology. 2006; 67:383-388. [PubMed: 16894096]

WHO/UNAIDS. Report on the Global AIDS Epidemic. 2008. Epub: http://www.unaids.org/en/ KnowledgeCentre/HIVData/GlobalReport/2008/2008_Global_report.asp

Williams PL, Marino M, Malee K, Brogly S, Hughes MD, Mofenson LM. Neurodevelopment and in utero antiretroviral exposure of HIV-exposed uninfected infants. Pediatrics. 2010; 125 :e250-e260. [PubMed: 20083530] 